\title{
Retinal Microvasculature Parameters with Optical Coherence Tomography Angiography and Mean Ocular Perfusion Pressure Changes in Mild Cognitive Impairment and Alzheimer's Disease
}

\section{Dandan Zhu}

Nanjing Drum Tower Hospital, the Affiliated Hospital of Nanjing University Medical School

Xun Liu

Nanjing Drum Tower Hospital, the Affiliated Hospital of Nanjing University Medical School

\section{Wenwen Zhang}

Nanjing Drum Tower Hospital, the Affiliated Hospital of Nanjing University Medical School

Jing Qiao

Nanjing Drum Tower Hospital, the Affiliated Hospital of Nanjing University Medical School

\section{Li Zhou}

Nanjing Drum Tower Hospital, the Affiliated Hospital of Nanjing University Medical School Zheng-Gao Xie ( $\nabla$ zgxie87@njglyy.com )

Nanjing Drum Tower Hospital, the Affiliated Hospital of Nanjing University Medical School

\section{Research Article}

Keywords: Alzheimer's disease, mild cognitive impairment, optical coherence tomography angiography, Mean arterial pressure, mean ocular perfusion pressure, Mini-Mental Status Examination

Posted Date: February 4th, 2022

DOl: https://doi.org/10.21203/rs.3.rs-1238950/v1

License: (c) (i) This work is licensed under a Creative Commons Attribution 4.0 International License. Read Full License 


\section{Abstract \\ Background}

To explore the ocular vascular variation involvement in mild cognitive impairment (MCl) and Alzheimer's disease (AD) cohorts. The measurement of the retinal vessel density was performed by spectral-domain optical coherence tomography angiography (OCTA).

\section{Methods}

This study including 24 eyes of normal cognitively individuals ( $\mathrm{NC}$ group), 23 eyes of $\mathrm{MCl}$ patients ( $\mathrm{MCl}$ group) and 11 eyes of AD patients (AD group) measured retinal vessel density with OCTA. Mean arterial pressure (MAP) and mean ocular perfusion pressure (MOPP) were calculated based on systemic blood pressure and intraocular pressure. We estimated the association between systemic and ocular vascular elements with cognitive assessment test (Mini-Mental Status Examination, MMSE) result by Pearson's correlation and linear regression model test.

\section{Results}

The retinal microvascular density were significantly lower in AD group compared to NC group while foveal avascular zone(FAZ) area were larger in AD group versus NC group. Both FAZ and vascular density parameters showed significant correlation with MMSE. After multivariate linear regression analysis, only FAZ and outer ring of optic nerve head were significantly associated MMSE. There was significant difference among three groups in MAP value while it was MOPP was found significant correlation with MMSE.

\section{Conclusion}

The OCTA parameters showed statistic difference between AD group and NC group. While only FAZ and outer ring of optic nerve head were significantly associated with estimated MMSE in multivariate linear regression analysis. So FAZ and outer ring of optic nerve head might be regarded as potential indicators for representing early sign of preclinical stage of AD.

\section{Introduction}

Alzheimer's disease (AD) is the most common form of dementia in western countries, affecting elderly people with irreversible cognitive decline and neurodegeneration disorders[1].The pathogenesis of $A D$ is characterized as the deposition of misfolded $\beta$-amyloid $(A \beta)$ and abnormal production of tau proteins in the brain[2]. Extensive evidence appears to suggest strong association between abnormal $A \beta$ accumulation and cerebrovascular pathologies, which are both related to the progression of cognitive impairment clinically in $A D$ patients[3]. While it may last for decades from the preclinical phases of $A D$ to the emergence of obvious clinical symptoms, the continuum of neurocognitive decline occurs along the spectrum ranging from normal cognition to mild cognitive impairment $(\mathrm{MCl})$ to dementia[4]. At present, there is no available treatment for AD; however cumulative evidence has supported the promising effect of medical intervention during the preclinical stage of $A D$ patients $[5,6]$. Therefore, 
the theme of how to assess and diagnose the precursor phase of AD has gained great interest from a massive number of researchers.

In terms of diagnostic criteria for screening cognitive impairment, several studies have demonstrated cognitive screening tools such as the Montreal Cognitive Assessment (MoCA) and the Mini-Mental Status Examination (MMSE) could be fundamental measurements for clinical evaluation of MCl cohorts[7]. Nevertheless, due to the inherent limitations of the MMSE and the MoCA, figuring out an alternative to detect cognitive deficits accurately is crucial. Nowadays, rapid advances in magnetic resonance imaging (MRI) and positron emission tomography (PET) scanning have provided precise evidence of $A \beta$ and tau deposition in $\mathrm{MCl}$ or $A D$ patients[8]. Besides that, characteristic biomarkers in peripheral blood or cerebrospinal fluid (CSF) has provided robust protein and inflammation network associated with the pathogenesis of $A D[9]$. However, all these examinations discussed above have limitations for being invasive or expensive and are not practical in large-scale screening for early diagnosing $\mathrm{MCl}$ or $\mathrm{AD}$. Thus, noninvasive and precise biomarkers are specially needed in clinical work as options for early diagnosis or therapeutic monitoring of $A D$ patients.

The retina and optic nerve are regarded as the 'window' of observing the central nervous system, since the retina contains similar functional and anatomical features with the cerebral vasculature[10,11]. For this reason, a number of researchers have proposed the hypothesis that the cerebral neurodegenerative disorders might play a pivotal role in the retinal pathological alterations. With the optical instrument including Fundus photography and spectral domain optical coherence tomography (SD-OCT), many studies have demonstrated retinal vascular morphology impairment and retinal thickness decrease in $A D$ and $M C I$ patients[12, 13]. With the advancement of retinal imaging techniques, optical coherence tomography angiography(OCTA) could provide high-resolution image rapidly and noninvasively and pose a tantalizing opportunity to gain more insights on retinal capillaries pathology.

This would be a valuable tool in exploring the cerebral neurodegenerative disorders[14]. Based on vascular alterations disclosed by using OCTA in patients with AD, several studies have reported that the retinal capillary vessel density is reduced while the foveal avascular zone (FAZ) is enlarged in comparison to the control group[15, 16].

Except for the detection of OCTA signals in AD, there has been an increase body of research on the relationship between systematic blood flow variability and AD. Till now, it remains unknown as to whether the effect of increased blood pressure(BP) contributes to the pathogenesis of $A D$ in vivo[17]. While there has been evidence of systemic hypertension can cause damage to the microvascular net of the optic disk[18]. And mean ocular perfusion pressure (MOPP), which directly related to blood pressure (BP) and intraocular pressure(IOP) level has been found increased in hypertension patients compared to the controls. So there are several reasons to explore the search for MOPP changes in AD pathological changes.

Herein, the focus of this study is to provide further evidence and evaluate the effect of OCTA predictors and blood flow indicators including mean arterial pressure (MAP) and mean ocular perfusion pressure (MOPP) as biomarkers and screening modalities for early definition of clinical AD.

\section{Methods}

\section{Study participants and Recruitments}


For this cross-sectional study, we evaluated 24 normal cognitively individuals (NC group), $23 \mathrm{MCl}$ patients (MCl group), 11 AD patients (AD group). The study was conducted from September 2019 to October 2020. All the subjects were diagnosed and recruited from the department of neurology in Nanjing Drum Tower Hospital. All cases underwent MoCA and MMSE tests for assessment of neuropsychological state, MRI scanning for excluding other brain diseases. All cases underwent ophthalmological examinations including best corrected visual acuity (BCVA), intraocular pressure(IOP), slit-lamp biomicroscopy and dilated fundus examination. Exclusion criteria included (1) refusal or unable to give informed consent; (2) metabolic disorders such as diabetes with uncontrollable glucose levels or obesity; (3) hypertension or hypotension with uncontrollable blood pressure level; (4) other neurodegenerative or optic nerve degenerative diseases such as Parkinson's disease, multiple sclerosis and non-arteritic anterior ischemic optic neuropathy (NA-AION); (5) other ocular diseases such as to significant media opacities, serious refractive errors with poor corrected vision glaucoma, macular disorders; poor fixation; any history of retinal surgery or treatment.

All the subjects were taken systolic blood pressure (SBP) and diastolic blood pressure (DBP) measurements, based on SBP and DBP levels, mean arterial pressure (MAP) and mean ocular perfusion pressure (MOPP) were calculated respectively by using the formula[19]:

\section{$M A P=1 / 3 * S B P+2 / 3 D B P ; M O P P=2 / 3 M A P-I O P$ Optical coherence tomography angiography (OCTA)}

OCTA image was selected from the right eye of every patient and the control. Once the right eye image quality was not up to the standard, then the left eye image was chosen. The chosen OCTA image should meet the signal quality criteria on a 10-degree scale and each measurement image with signal quality (SQ) of 6 or more.

The macular and the optic disc microvasculature images was carried out with the Cirrus HD-October 5000 instrument with AngioPlex (Carl Zeiss Meditec; 10.0 software version). For macular scope, a $4.5 \cdot 4.5 \mathrm{~mm}^{2} \mathrm{area}^{2}$ vessel densities centered on macular region was automated chosen and measured one value for the inner ring (1-3 $\mathrm{mm}$ around the fovea), the outer ring ( $3-6 \mathrm{~mm}$ around the fovea), and the center of the fovea ( $1 \mathrm{~mm}$ around the fovea) as well as the value of FAZ. For optic disc scope, a 3-6 $\mathrm{mm}$ ring around the optic nerve head (ONH) region was automated measured the radial peripapillary capillary (RPC) vessels density and documented as the inner ring, the outer ring and the center ring.

Other parameters related to $\mathrm{ONH}$ including retinal nerve fiber layer (RNFL) thickness; rim /disc area; average cup-todisc ratio (C/D); vertical C/D and cup volume were calculated automatically (Figure 1).

\section{Statistical analysis}

Statistical analyses were performed using SPSS for Windows statistical software (ver. 17.0; SPSS Inc., Chicago, IL, USA). Data are presented as mean \pm SD for continuous variables and as percentages for categorical variables. ANOVA test was performed to determine the differences among categorical variables. Mean values data did not show a Gaussian distribution, so it was compared by the Kruskal-Wallis test and Mann-Whitney u test. Pearson's coefficient was used to check correlation between retinal morphologic parameters (FAZ, macular and ONH region) and cognitive assessment test (MMSE) and ocular perfusion pressure (MOPP), respectively. And then a univariate linear regression model and a multiple linear regression model was used to analyze the strength of the association 
of MMSE and OCTA parameters; MMSE and blood perfusion parameters. $\mathrm{P}<0.05$ was interpreted as statistically significant.

\section{Results}

Baseline demographics and MMSE/ MoCA scores of the three groups

Our study initially recruited eighty participants. Two subjects were excluded for diagnosed glaucoma; Eight subjects were excluded for undiagnosed epi-retinal membrane; Twelve subjects were excluded for poor fixation or SQ<6 with incomplete OCTA results. Finally, 24 eyes of 24 healthy controls, 23 eyes of $\mathrm{MCl}$ patients and 11AD patients were enrolled in the present study. The baseline characteristics of participants recruited in the study are summarized in Table 1. There was no statistically significant difference in gender, age, BCVA, IOP, SBP and DBP among the three groups $(P>0.05)$. Both the $A D$ group and $\mathrm{MCl}$ group had lower scores than the $\mathrm{NC}$ group in MMSE and MoCA test $(P<0.05)$. Interestingly, there was no statistically significant difference in MOPP while the distribution of MAP was higher in the $A D$ group and $\mathrm{MCl}$ group than the $\mathrm{NC}$ group $(\mathrm{P}<0.05)$.

Table1. Baseline demographics and MMSE/ MoCA scores of the three groups

\begin{tabular}{|lllll|}
\hline Parameter & $\mathrm{NC}(\mathrm{n}=24)$ & $\mathrm{MCl}(\mathrm{n}=23)$ & $\mathrm{AD}(\mathrm{n}=11)$ & P value \\
\hline Gender(M/F) & $9 / 159$ & $7 / 16$ & $4 / 7$ & 0.97 \\
\hline Age(years) & $62.96 \pm 5.81$ & $67.61 \pm 9.34$ & $66.91 \pm 8.17$ & 0.11 \\
\hline BCVA(logMAR) & $0.22 \pm 0.08$ & $0.26 \pm 0.1$ & $0.26 \pm 0.09$ & 0.29 \\
\hline IOP(mmHg) & $14.29 \pm 3.06$ & $14.78 \pm 2.37$ & $15.82+3.06$ & 0.23 \\
\hline SBP(mmHg) & $133.1 \pm 7.66$ & $136.5 \pm 6.40$ & $136.6 \pm 4.59$ & 0.17 \\
\hline DBP(mmHg) & $87.79 \pm 6.41$ & $90.96 .7 \pm 3.76$ & $91.45 \pm 5.80$ & 0.07 \\
\hline MAP(mmHg) & $102.9 \pm 4.44$ & $106.1 \pm 3.07$ & $106.5 \pm 3.98$ & $0.01 *$ \\
\hline MOPP(mmHg) & $54.39 \pm 4.07$ & $55.62 \pm 2.92$ & $54.01 \pm 3.76$ & 0.37 \\
\hline MMSE(score) & $28.79 \pm 0.98$ & $26.87 \pm 2.16$ & $17.82 \pm 4.90$ & $<0.00^{*}$ \\
\hline MoCA(score) & $28.04(25.25,28)$ & $21.61(20,23)$ & $11.42(8.25,14.25)$ & $<0.00^{*}$ \\
\hline
\end{tabular}

${ }^{*} \mathrm{p}<0.05$

\section{Comparison of peripapillary perfusion density and microvascular density among the three groups}

On OCTA examination, comparison of mean value of peripapillary perfusion density and microvascular density in each group are listed in Table 2. Both peripapillary perfusion density and microvascular density decreased significantly in $A D$ group than in $N C$ group $(P<0.05)$. The comparison of peripapillary perfusion density and microvascular density analyses revealed a significant

loss in sections including outer ring of macula, central of optic nerve head, inner ring of optic nerve head, outer ring of optic nerve head in $\mathrm{MCl}$ group than in $\mathrm{NC}$ group $(\mathrm{P}<0.05)$. The value of microvascular density in sections including central of macula, inner ring of macula and FAZ showed no statistically difference between $\mathrm{MCl}$ group 
and NC group $(P>0.05)$. The section of inner ring/ outer ring of macula and inner ring/ outer ring of optic nerve head density decreased significantly in $A D$ group than in $\mathrm{MCl}$ group $(P<0.05)$. In terms of $F A Z$, the FAZ area in $A D$ group was the largest than the other two groups. Besides that, there was no statistical differences between $\mathrm{MCl}$ group and NC group in FAZ area comparison.

Table 2. Comparison of PPD and MVD among the three groups

\begin{tabular}{|c|c|c|c|c|c|c|c|}
\hline \multicolumn{4}{|c|}{ PPD and MVD } & \multicolumn{4}{|l|}{$P$ value } \\
\hline Parameter & NC & $\mathrm{MCl}$ & $A D$ & total & $\begin{array}{l}A D \\
\text { versus } \\
\text { NC }\end{array}$ & $\begin{array}{l}A D \\
\text { versus } \\
\mathrm{MCl}\end{array}$ & $\begin{array}{l}\mathrm{MCl} \\
\text { versus } \\
\mathrm{NC}\end{array}$ \\
\hline $\begin{array}{l}\text { Central of } \\
\text { macula }\end{array}$ & $8.04 \pm 3.28$ & $6.6 \pm 2.13$ & $5.43 \pm 1.96$ & $0.02^{\star}$ & $0.02^{\star}$ & 0.13 & 0.08 \\
\hline $\begin{array}{l}\text { Inner ring } \\
\text { of macula }\end{array}$ & $15.9 \pm 2.75$ & $15.83 \pm 2.16$ & $9.08 \pm 2.30$ & $<0.00^{*}$ & $<0.00 *$ & $<0.00^{*}$ & 0.88 \\
\hline $\begin{array}{l}\text { Outer ring } \\
\text { of macula }\end{array}$ & $17.98(17.28,18.83)$ & $14.9(12.9,16.4)$ & $11.15(9.7,12.7)$ & $<0.00 *$ & $<0.00 *$ & $<0.00 *$ & $<0.00^{\star}$ \\
\hline $\begin{array}{l}\text { Central of } \\
\text { ONH }\end{array}$ & $2.33 \pm 1.26$ & $1.45 \pm 1.15$ & $1.05 \pm 0.79$ & $0.01^{\star}$ & $0.01^{\star}$ & 0.3 & $0.02^{*}$ \\
\hline $\begin{array}{l}\text { Inner ring } \\
\text { of ONH }\end{array}$ & 18.38(17.85,18.9ه & $15.96(14.4,16.9)$ & $10.37(8.9,13)$ & $<0.00 *$ & $<0.00 *$ & $<0.00 *$ & $<0.00^{*}$ \\
\hline $\begin{array}{l}\text { Outer ring } \\
\text { of ONH }\end{array}$ & $18.83 \pm 0.65$ & $17.31 \pm 1.10$ & $11.09 \pm 3.04$ & $<0.00 *$ & $<0.00 *$ & $<0.00 *$ & $<0.00^{\star}$ \\
\hline FAZ & $0.21 \pm 0.09$ & $0.26 \pm 0.10$ & $0.38 \pm 0.11$ & $0.00^{\star}$ & $0.001 *$ & $0.01 *$ & 0.41 \\
\hline
\end{tabular}

${ }^{*} \mathrm{p}<0.05 ; \mathrm{ONH}$ : optic nerve head; PPD:peripapillary perfusion density; MVD: microvascular density

\section{Correlation of perfusion density and vascular density with blood flow indicators and MMSE}

Peripapillary perfusion density and microvascular density showed a significant correlation with MMSE in section including inner ring/ outer ring of macula and inner ring/ outer ring of optic nerve head, but not in central of macula and central of optic nerve head (Table 3). FAZ also exhibited a significant correlation with MMSE ( $r=-0.53$, $\mathrm{p}<0.0001$ ) (Figure 2). As to the correlation between blood flow indicators and MMSE, MOPP presented a statistically significant result $(r=-0.27, p=0.04)$ while MAP might not correlate with MMSE.

Table 3. Correlation of perfusion density and vascular density with blood flow indicators and MMSE 


\begin{tabular}{|llll|}
\hline Parameter & $\mathbf{r}$ & $95 \% \mathrm{Cl}$ & P value \\
\hline FAZ & -0.53 & -0.69 to- 0.31 & $<0.00^{\star}$ \\
\hline Central of macula & 0.13 & -0.132 to 0.37 & 0.34 \\
\hline Inner ring of macula & 0.38 & 0.13 to 0.58 & $0.00^{\star}$ \\
\hline Outer ring of macula & 0.63 & 0.44 to 0.76 & $<0.00^{\star}$ \\
\hline Central of ONH & 0.24 & -0.02 to 0.47 & 0.07 \\
\hline Inner ring of ONH & 0.71 & 0.55 to 0.82 & $<0.00^{\star}$ \\
\hline Outer ring of ONH & 0.75 & 0.62 to 0.85 & $<0.00^{\star}$ \\
\hline MOPP & -0.27 & -0.49 to-0.01 & $0.04 *$ \\
\hline MAP & -0.25 & -0.48 to 0.01 & 0.06 \\
\hline
\end{tabular}

${ }^{*} \mathrm{p}<0.05$

\section{The strength of the association between risk indicators and MMSE}

Nine Parameter s were analyzed by using univariate and multivariate linear regression model of MMSE (table 4). Results from the univariate linear regression model suggested that FAZ ( $95 \% \mathrm{Cl}-50.4$ to-19.16, $p<0.00)$, inner ring of macula (95\% Cl 0.51to1.07, p<0.00), outer ring of macula (95\% Cl 1.07to2.14, $\mathrm{p}<0.00)$, inner ring of $\mathrm{ONH}(95 \% \mathrm{Cl}$ 0.74 to1.27, $p<0.00)$, outer ring of $\mathrm{ONH}(95 \% \mathrm{Cl} 0.87$ to1.39, $\mathrm{p}<0.00)$ and MOPP $(95 \% \mathrm{Cl}-0.4$ to-0.01, $\mathrm{p}=0.04)$ were significantly associated MMSE. In multivariable linear regression model, only FAZ $(\beta=0.24, p=0.02)$ and outer ring of $\mathrm{ONH}(\beta=0.33, p=0.03)$ were significantly associated with MMSE.

Table 4. Linear regression analysis for association between risk indicators and MMSE

\begin{tabular}{|lllll|}
\hline Parameter & Univariate & & \multicolumn{2}{l|}{ Multivariate } \\
\hline FAZ & $95 \% \mathrm{Cl}$ & P value & Beta & P value \\
\hline Central of macula & -0.03 to0.86 & 0.07 & 0.01 & 0.96 \\
\hline Inner ring of macula & 0.51 to 1.07 & $<0.00 *$ & 0.15 & 0.22 \\
\hline Outer ring of macula & 1.071 to2.14 & $<0.00 *$ & 0.08 & 0.61 \\
\hline Central of ONH & -0.09 to 1.92 & 0.07 & 0.02 & 0.85 \\
\hline Inner ring of ONH & 0.74 to1.27 & $<0.00 *$ & 0.22 & 0.16 \\
\hline Outer ring of ONH & 0.87 to 1.39 & $<0.00 *$ & 0.33 & $0.03 *$ \\
\hline MOPP & -0.54 to-0.01 & $0.04 *$ & 0.05 & 0.59 \\
\hline MAP & -0.59 to 0.01 & 0.06 & 0.01 & 0.89 \\
\hline
\end{tabular}




\section{Discussion}

In this study, we compared retinal peripapillary perfusion density and microvascular density alterations by OCTA in $\mathrm{AD}$ and $\mathrm{MCl}$ patients with normal cognitively subjects. Besides that, we also estimated the effect of MAP and MOPP to interpret the progression of $A D$, which could be considered as confounding factors. Our data indicated that macular parameters (inner ring of macula, outer ring of macula), $\mathrm{ONH}$ parameters (inner ring of ONH, outer ring of $\mathrm{ONH}$ ) and FAZ presented significant correlation with MMSE while the central section of macular and $\mathrm{ONH}$ presented no significant correlation with MMSE. In terms of MOPP and MAP, although the value of MAP showed significant difference among three groups, MAP showed no significant correlation with MMSE in Pearson's and linear regression analyses. On the other hand, the value of MOPP showed no significant difference among three groups, which showed significant correlation with MMSE in Pearson's and univariate linear regression analyses.

Studies from the association between elevated blood pressure (BP) and AD pathology indicated that the impact of $\mathrm{BP}$ has been still controversial. A number of evidence conducted that there was no association between late life hypertension and brain $A \beta$ deposition[20-22]. However, some conflictual findings revealed that hypertension was associated with accelerated $A \beta$ accumulation[23]. Those variable results might be explained by findings from the following aspects: some data came from normal cognitively people and some research studied patients with reduced cognitive ability. Besides that, the diagnosis and measurement of hypertension also differed in those studies. Given the heterogeneity between studies, it should be difficult to make a meaningful analysis for the association between blood pressure and AD. The present study was partly consistent with previous research, reporting that the statistical difference in MAP did not markedly affect the association between MMSE and MAP. While the value of MOPP has shown different results: MOPP showed apparent relevance with MMSE although no significant difference was reached in primary values. One explanation for changes in MAP was that MAP depended on SBP and DBP regulation while MOPP depended on measurement of MAP and IOP, so MOPP might be more ideal than MAP to estimate the relevance between ocular vascular perfusion and the progression of AD. To the best of our knowledge, there has been few studies on changes of MOPP in patients with worsen cognitive decline. The majority of those research focused on the relationship between MOPP fluctuation and ocular ischemic disease with the optic nerve defect including diabetic retinopathy, glaucoma, non-arteritic anterior ischemic optic neuropathy (NAAION) [24-26]. MOPP has been shown strong association with the prevalence of those ocular disease. Thus MOPP might offer additional information to estimate the risk of cognitive decline and prediction of rates of neurodegeneration worsening.

A number of studies have also found that the presence of OCTA changes in patients developing neurodegenerative damage. Previous analysis has reported that the area of the FAZ was much larger in $A D$ or $\mathrm{MCI}$ patients than the controls[15, 16, 27]. Our OCTA data demonstrated a markedly enlargement of the FAZ in AD cohorts, which is in line with previous reports. Besides, we noted that the area of FAZ was much greater in AD group than the $\mathrm{MCl}$ group, while we found no significant difference in FAZ area between $\mathrm{MCl}$ group and $\mathrm{NC}$ group. The enlargement of the FAZ has been regarded as an important signal for retinal capillary occlusion and retinal ischemia[28], Furthermore, we exhibited a significant correlation between FAZ and MMSE, so our data suggested that FAZ size might be an indicator for progression of cognitive impairment. Whereas Wu et al found significant difference in $\mathrm{FAZ}$ area in $\mathrm{MCl}$ patients comparing with control group, which is inconsistent with our findings[27]. There may be various possible explanations for this discrepancy. First, the differences may be attributable to the small sample size, and the area of FAZ varies considerably between individuals in the study. Second, data obtained by different OCTA devices may not have good consistency due to various algorithms. 
In terms of other OCTA data, previous studies demonstrated a decrease in retinal vessel density and increase in FAZ area in $A D$ patients $[15,29]$. Our observation exhibited similar differences in vessel densities centered on macular region in the dementia phase of $\mathrm{MCl}$ and $\mathrm{AD}$ patients. Based on our findings, we found the obvious decrease of vessel density in all retinal areas (center/inner/outer ring of the macula, center/inner/outer around the $\mathrm{ONH}$ ) in $\mathrm{AD}$ group compared with the $\mathrm{NC}$ group. In contrast to the reduction of vessel density in all retinal areas, some researcher indicated the opposite result: they found vessel density in all retinal areas statistically significant higher in $A D$ patients than the control[27]. We assessed the explanation for those unexpected alterations might lie in the inflammatory reaction in the development of $A D$ accompanied with $A \beta$ accumulation. Supposing that inflammatory process in the onset stage of $A D$ with ischemic and hypoxic damage might result in retinal vasoconstriction increasing[30], which would be detected by OCTA and documented as visible retinal vessel density. With the progress of $A D$, chronic inflammation and $A \beta$ accumulation might cause loss of retinal microvasculature and the decrease in vessel density[31]. Considering the significant differences in vessel density around macular and $\mathrm{ONH}$ between the cognitive impairment and the normal cognitive individuals, we also found correlations between MMSE and OCTA parameters, including FAZ, the inner/outer ring of the macula and the inner/outer ring of $\mathrm{ONH}$, while no significant correlation was found in center of macula and center of ONH area. The univariate linear regression analysis was similar with the Pearson's correlation data, except the center of macula and center of ONH area showed no association with MMSE, other OCTA parameters were correlated with MMSE significantly. However, only FAZ and outer ring of ONH showed statistical association with MMSE and the other OCTA parameters lost associations after multiple linear regression analysis. The possible explanations for the lack of this association might attribute to the distribution of the value of MMSE. Outcomes on calculation in MMSE and MoCA varied across individuals and placed a high load on personal abilities including language, education stratification[32]. Although MoCA test seemed to fare better than MMSE in detecting cognitive impairment clinically[33], the MoCA score in our study exhibited obvious fluctuations and hardly normalization, so we chose MMSE score to represent the dementia phrase.

To the best of our knowledge, few studies have investigated OCTA features and ocular perfusion pressure in cognitive impairment patients simultaneously. Our study characterized the thorough OCTA descriptive indicators in participants with early and development stage of cognitive impairment. We explored vascular involvement in AD development extensively by analyzing MOPP and MAP.

We acknowledge some limitations to this study. This was a single-center, cross-sectional trial with a limited number of samples. It is necessary to conduct a larger number of subjects and follow-up study to confirm our findings. And due to the algorithms of OCTA, artifacts cannot be avoided. Furthermore, this only measure the central foveal area of $4.5 \times 4.5 \mathrm{~mm}$, which may be limited in detecting early microvascular changes in outer region. PET scanning provided rather sensitive visualization of $A \beta$ accumulation in vivo, which might be compared with OCTA parameters and MOPP in the future.

In summary, we found a significant lower retinal vessel density around macular and ONH regions in AD versus NC participants. We also discovered FAZ and outer ring of ONH showed strong association with MMSE score in multiple linear regression analysis, suggesting those two parameters could be possible biomarker for predicting the onset of AD clinically. No significant association was found between ocular perfusion indicators(MOPP/MAP) and MMSE in multiple linear regression analysis, however, we confirmed the significant difference among three groups and MOPP showed moderate association with MMSE in Pearson correlation and univariate linear regression analysis. Further in vivo studies are needed to understand the pathologic mechanistic insights into ocular vascular involvement in AD clinical process. 


\section{Abbreviations}

Alzheimer's disease (AD); mild cognitive impairment (MCl); optical coherence tomography angiography (OCTA); Mean arterial pressure (MAP) ; Mean ocular perfusion pressure (MOPP); Cognitive assessment test (Mini-Mental Status Examination, MMSE) ; foveal avascular zone(FAZ); Montreal Cognitive Assessment (MoCA); optic nerve head $(\mathrm{ONH})$

\section{Declarations}

\section{Ethics approval and consent to participate}

All the subjects were diagnosed and recruited from the department of neurology in Nanjing Drum Tower Hospital. The study was performed in accordance with the Declaration of Helsinki', and the study was approved by the Nanjing Drum Tower Hospital Ethics Committee. Written Informed Consent' from all the patients before participating for the study.

\section{Consent for publication}

Not applicable.

\section{Availability of data and materials}

All data generated or analysed during this study are included in this published article.

\section{Competing interests}

The authors declare that they have no competing interests.

\section{Funding}

Not applicable.

\section{Authors' contributions}

ZDD and LX performed the experiments, analyzed the data and ZDD was a major contributor in writing the manuscript. ZWW analyzed and interpreted the patient data. ZL and QJ completed the ocular examinations, XZG designed and supervised the study. All authors read and approved the final manuscript.

\section{Acknowledgments}

We thank all the patients who contributed to this study.

\section{References}

1. Blennow K, de Leon MJ, Zetterberg H: Alzheimer's disease. Lancet (London, England) 2006, 368(9533):387403.

2. Wang J, Gu BJ, Masters CL, Wang YJ: A systemic view of Alzheimer disease - insights from amyloid- $\beta$ metabolism beyond the brain. Nature reviews Neurology 2017, 13(10):612-623. 
3. Rabin JS, Yang HS, Schultz AP, Hanseeuw BJ, Hedden T, Viswanathan A, Gatchel JR, Marshall GA, Kilpatrick E, Klein $\mathrm{H}$ et al: Vascular Risk and $\beta$-Amyloid Are Synergistically Associated with Cortical Tau. Annals of neurology 2019, 85(2):272-279.

4. Anderson ND: State of the science on mild cognitive impairment (MCl). CNS spectrums 2019, 24(1):78-87.

5. Abeysinghe A, Deshapriya R, Udawatte C: Alzheimer's disease; a review of the pathophysiological basis and therapeutic interventions. Life sciences 2020, 256:117996.

6. Yiannopoulou KG, Papageorgiou SG: Current and Future Treatments in Alzheimer Disease: An Update. Journal of central nervous system disease 2020, 12:1179573520907397.

7. Ciesielska N, Sokołowski R, Mazur E, Podhorecka M, Polak-Szabela A, Kędziora-Kornatowska K: Is the Montreal Cognitive Assessment (MoCA) test better suited than the Mini-Mental State Examination (MMSE) in mild cognitive impairment $(\mathrm{MCl})$ detection among people aged over 60? Meta-analysis. Psychiatria polska 2016, 50(5):1039-1052.

8. Nägga K, Gustavsson AM, Stomrud E, Lindqvist D, van Westen D, Blennow K, Zetterberg H, Melander O, Hansson O: Increased midlife triglycerides predict brain $\beta$-amyloid and tau pathology 20 years later. Neurology 2018, 90(1):e73-e81.

9. Johnson ECB, Dammer EB, Duong DM, Ping L, Zhou M, Yin L, Higginbotham LA, Guajardo A, White B, Troncoso $\mathrm{JC}$ et al: Large-scale proteomic analysis of Alzheimer's disease brain and cerebrospinal fluid reveals early changes in energy metabolism associated with microglia and astrocyte activation. Nature medicine 2020, 26(5):769-780.

10. London A, Benhar I, Schwartz M: The retina as a window to the brain-from eye research to CNS disorders. Nature reviews Neurology 2013, 9(1):44-53.

11. Williams MA, McGowan AJ, Cardwell CR, Cheung CY, Craig D, Passmore P, Silvestri G, Maxwell AP, McKay GJ: Retinal microvascular network attenuation in Alzheimer's disease. Alzheimer's \& dementia (Amsterdam, Netherlands) 2015, 1(2):229-235.

12. Snyder PJ, Johnson LN, Lim YY, Santos CY, Alber J, Maruff P, Fernández B: Nonvascular retinal imaging markers of preclinical Alzheimer's disease. Alzheimer's \& dementia (Amsterdam, Netherlands) 2016, 4:169-178.

13. Kashani AH, Asanad S, Chan JW, Singer MB, Zhang J, Sharifi M, Khansari MM, Abdolahi F, Shi Y, Biffi A et al: Past, present and future role of retinal imaging in neurodegenerative disease. Progress in retinal and eye research 2021, 83:100938.

14. Tsokolas G, Tsaousis KT, Diakonis VF, Matsou A, Tyradellis S: Optical Coherence Tomography Angiography in Neurodegenerative Diseases: A Review. Eye and brain 2020, 12:73-87.

15. Jiang H, Wei Y, Shi Y, Wright CB, Sun X, Gregori G, Zheng F, Vanner EA, Lam BL, Rundek T et al: Altered Macular Microvasculature in Mild Cognitive Impairment and Alzheimer Disease. Journal of neuro-ophthalmology : the official journal of the North American Neuro-Ophthalmology Society 2018, 38(3):292-298.

16. Bulut M, Kurtuluş F, Gözkaya O, Erol MK, Cengiz A, Akıdan M, Yaman A: Evaluation of optical coherence tomography angiographic findings in Alzheimer's type dementia. The British journal of ophthalmology 2018, 102(2):233-237.

17. Lennon MJ, Koncz R, Sachdev PS: Hypertension and Alzheimer's disease: is the picture any clearer? Current opinion in psychiatry 2021, 34(2):142-148.

18. Kanadani FN, Figueiredo CR, Miranda RM, Cunha PL, TC MK, Dorairaj S: Ocular Perfusion Pressure and Pulsatile Ocular Blood Flow in Normal and Systemic Hypertensive Patients. Journal of current glaucoma 
practice $2015,9(1): 16-19$.

19. Yun YI, Kim YW, Lim HB, Lee DH, Kim JH, Oh BL, Jeoung JW, Park KH: Peripapillary vessel parameters and mean ocular perfusion pressure in young healthy eyes: OCT angiography study. The British journal of ophthalmology 2021, 105(6):862-868.

20. Lane CA, Barnes J, Nicholas JM, Sudre CH, Cash DM, Parker TD, Malone IB, Lu K, James SN, Keshavan A et al: Associations between blood pressure across adulthood and late-life brain structure and pathology in the neuroscience substudy of the 1946 British birth cohort (Insight 46): an epidemiological study. The Lancet Neurology 2019, 18(10):942-952.

21. Kemppainen N, Johansson J, Teuho J, Parkkola R, Joutsa J, Ngandu T, Solomon A, Stephen R, Liu Y, Hänninen $\mathrm{T}$ et al: Brain amyloid load and its associations with cognition and vascular risk factors in FINGER Study. Neurology 2018, 90(3):e206-e213.

22. Vemuri P, Lesnick TG, Przybelski SA, Knopman DS, Lowe VJ, Graff-Radford J, Roberts RO, Mielke MM, Machulda MM, Petersen RC et al: Age, vascular health, and Alzheimer disease biomarkers in an elderly sample. Annals of neurology 2017, 82(5):706-718.

23. Gomez G, Beason-Held LL, Bilgel M, An Y, Wong DF, Studenski S, Ferrucci L, Resnick SM: Metabolic Syndrome and Amyloid Accumulation in the Aging Brain. Journal of Alzheimer's disease : JAD 2018, 65(2):629-639.

24. Zhai G, Lin Z, Wang FH, Wang Y, Li D, Wen L, Ding XX, Jiang J, Feng KM, Liang YB et al: Association between Mean Ocular Perfusion Pressure and Diabetic Retinopathy in a Northeastern Chinese Population. Biomedical and environmental sciences : BES 2020, 33(9):701-707.

25. Gore V, Shah P, Kanhere M, Gore S: Relationship between optical perfusion pressure and systemic blood pressure on glaucoma: Case-control study. Oman journal of ophthalmology 2019, 12(3):150-155.

26. Lacharme T, Almanjoumi A, Aptel F, Khayi H, Pepin JL, Baguet JP, Romanet JP, Chiquet C: Twenty-four-hour rhythm of ocular perfusion pressure in non-arteritic anterior ischaemic optic neuropathy. Acta ophthalmologica 2014, 92(5):e346-352.

27. Wu J, Zhang X, Azhati G, Li T, Xu G, Liu F: Retinal microvascular attenuation in mental cognitive impairment and Alzheimer's disease by optical coherence tomography angiography. Acta ophthalmologica 2020, 98(6):e781-e787.

28. Kim K, Kim ES, Yu SY: Optical coherence tomography angiography analysis of foveal microvascular changes and inner retinal layer thinning in patients with diabetes. The British journal of ophthalmology 2018, 102(9):1226-1231.

29. Grewal DS, Polascik BW, Hoffmeyer GC, Fekrat S: Assessment of Differences in Retinal Microvasculature Using OCT Angiography in Alzheimer's Disease: A Twin Discordance Report. Ophthalmic surgery, lasers \& imaging retina 2018, 49(6):440-444.

30. Kinney JW, Bemiller SM, Murtishaw AS, Leisgang AM, Salazar AM, Lamb BT: Inflammation as a central mechanism in Alzheimer's disease. Alzheimer's \& dementia (New York, N Y) 2018, 4:575-590.

31. Sousa DC, Leal I, Moreira S, Dionísio P, Abegão Pinto L, Marques-Neves C: Hypoxia challenge test and retinal circulation changes - a study using ocular coherence tomography angiography. Acta ophthalmologica 2018, 96(3):e315-e319.

32. Khaw J, Subramaniam P, Abd Aziz NA, Ali Raymond A, Wan Zaidi WA, Ghazali SE: Current Update on the Clinical Utility of MMSE and MoCA for Stroke Patients in Asia: A Systematic Review. International journal of environmental research and public health 2021, 18(17). 
33. Trzepacz PT, Hochstetler H, Wang S, Walker B, Saykin AJ: Relationship between the Montreal Cognitive Assessment and Mini-mental State Examination for assessment of mild cognitive impairment in older adults. BMC geriatrics 2015, 15:107.

\section{Figures}
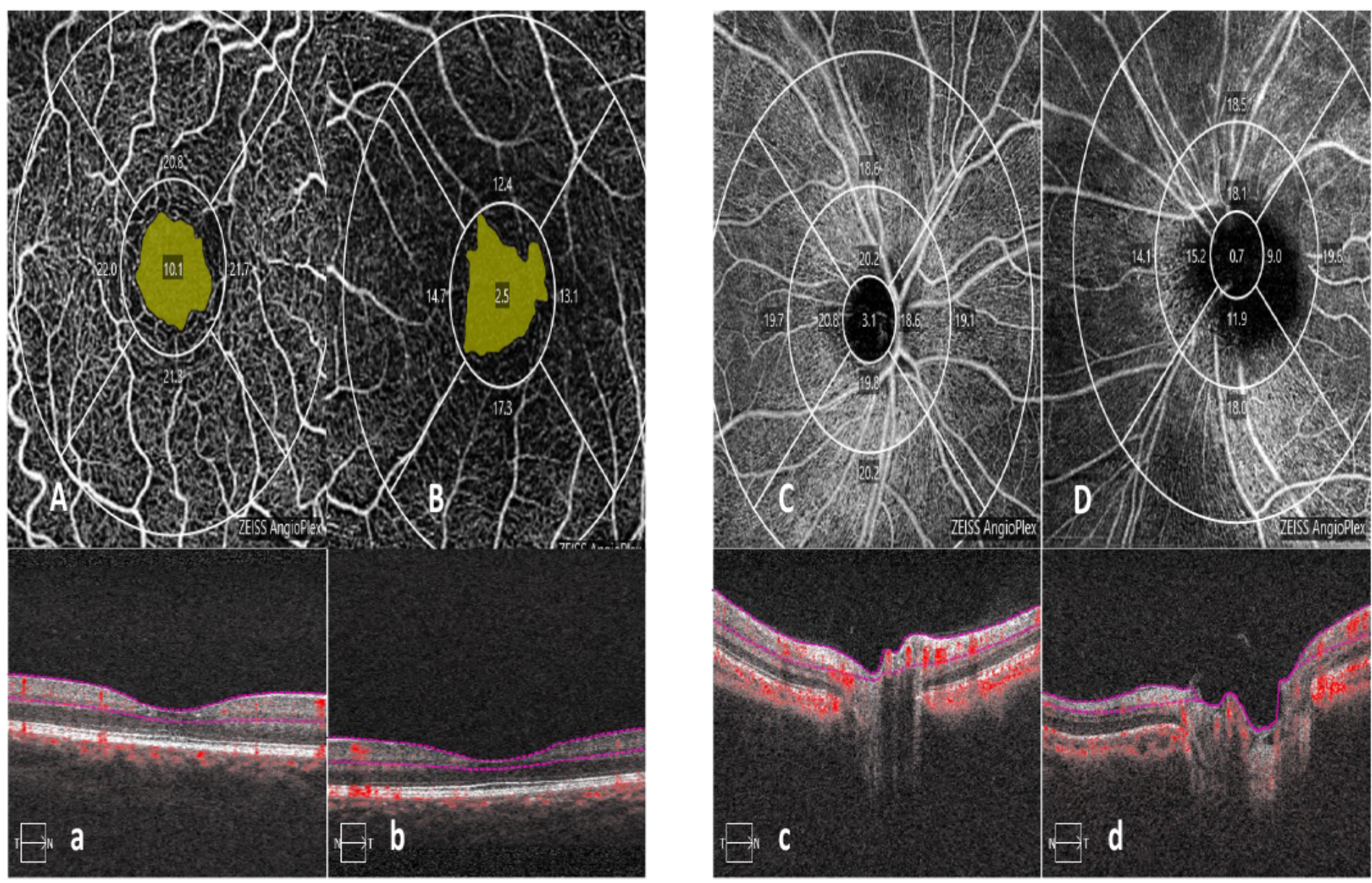

Figure 1

OCTA scans A: vascular density analysis of the macular region in NC group; the green area was automated FAZ measurement; a: between two red lines was corresponding to Figure 1A showing the location of internal limiting membrane-inner plexiform layer; $B$ : vascular density analysis of the macular region in AD group; the green area was automated FAZ measurement; b: corresponding to Figure 1B showing the location of internal limiting membraneinner plexiform layer; $\mathrm{C}$ : vascular density analysis of the $\mathrm{ONH}$ region in NC group; c: corresponding to Figure $1 \mathrm{C}$ showing the location of $\mathrm{ONH}$; $\mathrm{D}$ : vascular density analysis of the $\mathrm{ONH}$ region in $\mathrm{AD}$ group; $\mathrm{d}$ : corresponding to Figure 1D showing the location of $\mathrm{ONH}$ 


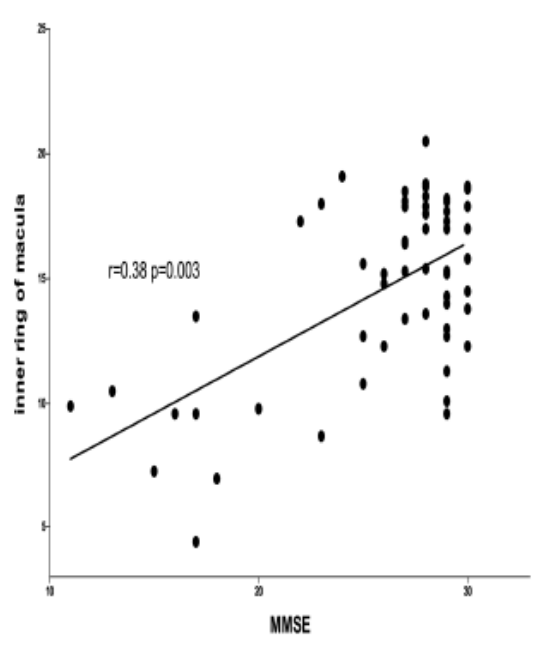

A

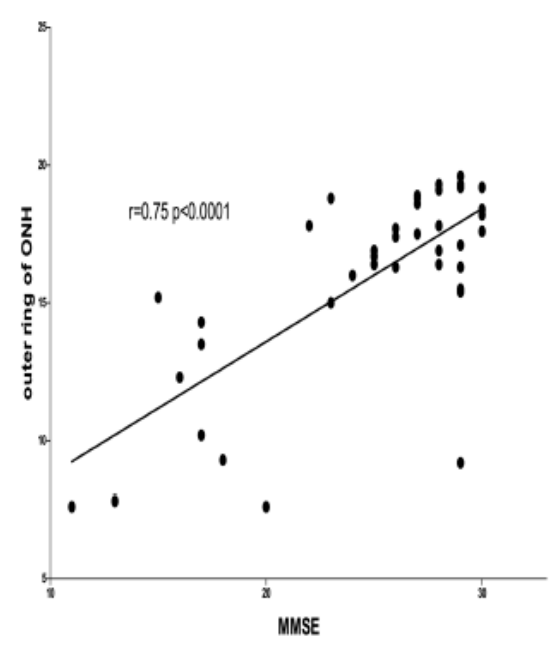

D

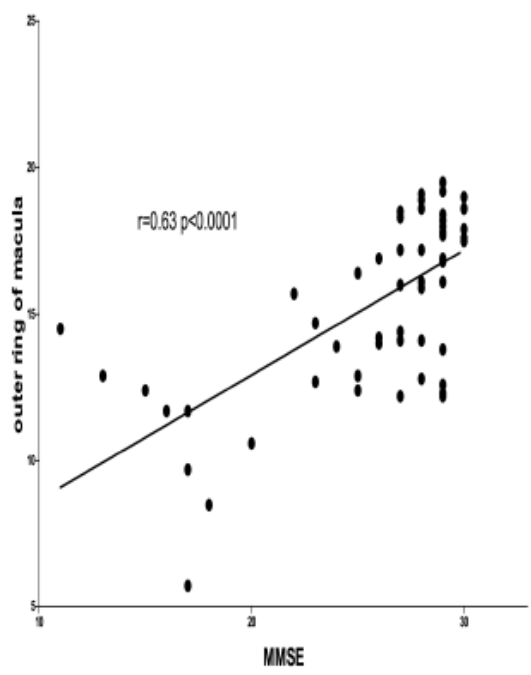

B

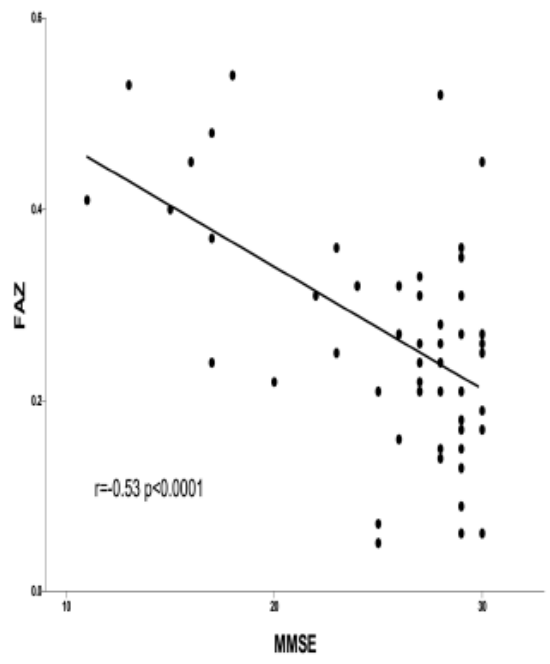

$E$

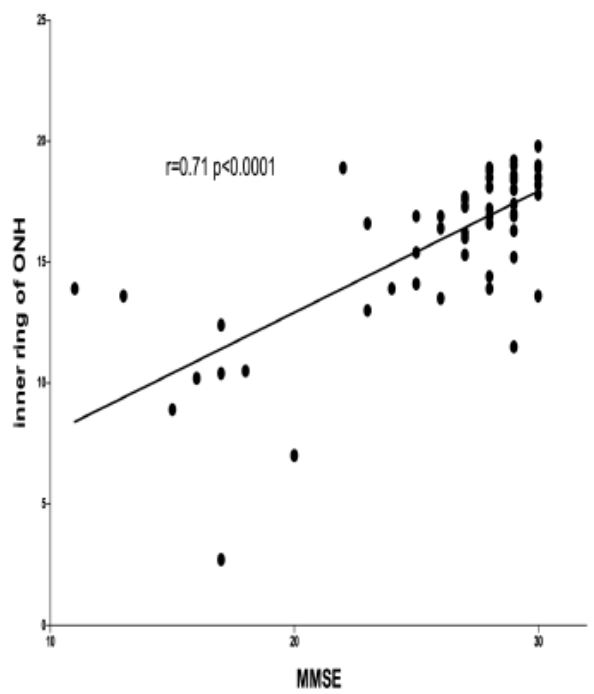

C

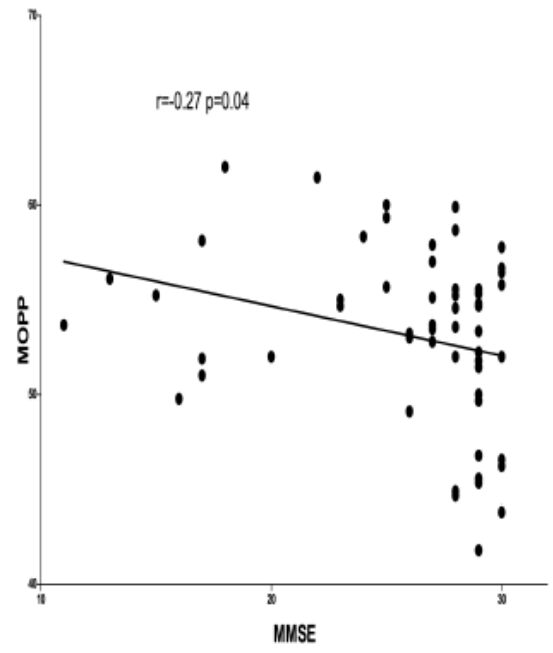

F

Figure 2

Correlation of perfusion density and vascular density with blood flow indicators and MMSE (A) inner ring of macular; (B) outer ring of macula ; (C) inner ring of $\mathrm{ONH}$; (D) outer ring of ONH; (E) FAZ; $(F)$ MOPP 